\title{
Viabilidade agroeconômica do consórcio de taro (Colocasia esculenta L.) e pepino em função do arranjo de plantas ${ }^{1}$
}

\author{
João Nacir Colombo ${ }^{2}$, Mário Puiatti ${ }^{3}$,Jaime Barros da Silva Filho ${ }^{4}$, Janiele Cássia Barbosa Vieira ${ }^{4}$, \\ Geovani do Carmo Copati da Silva ${ }^{3}$
}

10.1590/0034-737X201865010008

\begin{abstract}
RESUMO
A cultura do taro (Colocasia esculenta L.), cultivar Japonês, é bastante interessante para o cultivo consorciado, porque apresenta ciclo longo e rusticidade, em relação a fatores ambientais e biológicos adversos. Objetivou-se, com este trabalho, avaliar a viabilidade agronômica e a rentabilidade econômica do consórcio taro e pepino. O experimento foi conduzido a campo, de outubro de 2012 a junho de 2013. Constou de sete tratamentos, sendo o pepino, em três deles, cultivado na forma de consórcio com o taro, com 1 planta a cada $30 \mathrm{~cm} ; 2$ plantas a cada $60 \mathrm{~cm}$, opostas entre si e opostas alternadas entre si nas linhas. Outros três tratamentos consistiram em cultivo do pepino solteiro, seguindo a mesma distribuição do consorciado. Para o último tratamento, foi utilizado o taro solteiro. O delineamento experimental foi o de blocos casualizados, com quatro repetições. Foram determinados os parâmetros de fluorescência da clorofila, produtividade das culturas e indicadores econômicos. Os consórcios favoreceram a produção de pepino, mas afetaram a do taro. Todos os consórcios foram agronomicamente viáveis, por apresentarem uso eficiente da terra acima da unidade, além de renda bruta superior ao do controle. Entretanto, por causa do baixo valor de mercado para o pepino, por ocasião da colheita, a renda líquida não diferiu da renda do monocultivo do taro. Os parâmetros da fluorescência da clorofila podem explicar, em parte, os menores valores de produção de rizomas de taro obtidos nos tratamentos em associação com pepino.
\end{abstract}

Palavras-chave: Cucumis sativus; rentabilidade; sustentabilidade; agricultura familiar.

\section{ABSTRACT}

\section{Agroeconomic viability of taro (Colocasia esculenta L.) and cucumber consortium in function of plant arrangement}

Taro culture presents hardiness in relation to adverse environmental and biological factors and long cycle, making it an interesting culture for intercropping. The objective of this work was to evaluate the agronomic viability and economic yield of taro intercropped (additive association) with cucumber. The experiment was conducted in the field from October 2012 to June 2013. It consisted of seven treatments, having cucumber cultivated, in three of them, in the form of consortium with one taro plant every $30 \mathrm{~cm}$; two plants each $60 \mathrm{~cm}$ opposite to each other, and alternatingly opposing each other in the lines. Three other treatments consisted of single cucumber cultivation, following the same distribution of the consortium. For the last treatment, taro monoculture was used. The experiments were arranged in a randomized block design with four replications. We determined chlorophyll fluorescence parameters, crop productivity, and economic indicators. The intercropping favored cucumber production but affected taro production. All consortia were agronomically viable for presenting land use efficiency above the unit, as well as higher gross income than the control and early entry resources with the cucumber harvest; however, due to the low market value for cucumber at harvest, net income did not differ from taro monoculture. Chlorophyll fluorescence parameters may explain, in part, the lowest taro production values obtained in the treatments in association with cucumber.

Key words: Cucumis sativus; profitability; sustainability; family agriculture.

\footnotetext{
Submetido em 03/02/2015 e aprovado em 29/01/2018.

Trabalho financiado pelo CNPQ e FAPEMIG.

2 Instituto Federal do Espírito Santo, Campus Santa Teresa, Santa Teresa, Espírito Santo, Brasil. joaonacirc@yahoo.com.br

3 Universidade Federal de Viçosa, Departamento de Fitotecnia, Viçosa, Minas Gerais, Brasil. mpuiatti@ufv.br; geovani.silva@ufv.br

4 Universidade Federal de Viçosa, Departamento de Fitotecnia, Programa de Pós-graduação em Fitotecnia, Viçosa, Minas Gerais, Brasil. jaimeufv@gmail.com; jani cassia@yahoo.com.br *Autora correspondente: jani cassia@yahoo.com.br
} 


\section{INTRODUÇÃO}

No Brasil, existem 4.367.902 de estabelecimentos de agricultores familiares, o que representa $84,4 \%$ do total de estabelecimentos rurais brasileiros (IBGE, 2006). A agricultura familiar gera mais de $80 \%$ da ocupação no setor rural e responde por sete de cada dez empregos no campo e por cerca de $40 \%$ da produção agrícola brasileira. Em 2009, cerca de $60 \%$ dos alimentos que compuseram a cesta alimentar distribuída pela Conab originaram-se da agricultura familiar (CONAB, 2015). Esses números mostram a importância desse grupo de produtores para a agricultura e para a economia nacional. Todavia é preciso estimular esses agricultores, pesquisando e repassando tecnologias e informações que possam melhorar a renda da propriedade e, consequentemente, sua qualidade de vida e a de seus familiares.

Estudos sobre consorciação de culturas estão contribuindo para o encontro de futuros caminhos para a agricultura tradicional e para que se descubra o quão mais avançadas podem ser as tecnologias da consorciação em relação às dos monocultivos, quanto à sustentabilidade do ambiente, mediante a manutenção da biodiversidade, conservação do solo, reciclagem de nutrientes, controle de plantas daninhas, controle de pragas e doenças e aumento da produtividade (Innis, 1997).

Fatores como a identificação de cultivos e o ajuste de arranjos populacionais podem ser manipulados para melhorar o uso dos recursos naturais e a eficiência do consórcio de hortaliças (Barros Júnior et al., 2005). A cultura do taro apresenta rusticidade em relação a fatores ambientais e biológicos adversos, aspectos esses positivos em termos de cultivo alternativo para a agricultura familiar. Todavia, o ciclo cultural longo (cerca de nove meses), inviabiliza o seu cultivo em pequenas propriedades. Dessa forma, a consorciação do taro com outras culturas, que proporcionem colheitas antecipadas, seria uma opção interessante, por permitir a obtenção de renda ao longo do cultivo.

Trabalhos avaliando o sombreamento artificial do taro (Gondim et al., 2007 e Oliveira et al., 2011) demonstraram ser essa cultura tolerante ao sombreamento de 18 a $25 \%$, o que indicou que pode ser consorciada com hortaliças de maior porte.

Embora o taro apresente características morfológicas e fisiológicas que permitem a associação com outras espécies, pesquisas envolvendo especificamente consórcio do taro com outras hortaliças são relativamente poucas, dentre as quais temos: taro com milho doce (Puiatti et al., 2000); taro com alface (Heredia Zárate et al., 2005; 2007a); taro com chicória (Heredia Zárate et al., 2007b); taro com cenoura e alface (Heredia Zárate et al., 2006) e taro com feijão-vagem (Vieira et al., 2014).
O pepino (Cucumis sativus) é uma cucurbitácea cuja produção anual brasileira é de 215.117 toneladas, sendo produzidas, na região sudeste, 113.640 toneladas (IBGE, 2006). A espécie é adaptada ao cultivo sob temperaturas superiores a $20^{\circ} \mathrm{C}$, exigências semelhantes às da cultura do taro, aspecto esse que torna factível o cultivo dessas espécies em associação. Todavia, além da escolha das espécies e dos cultivares, os arranjos espaciais são importantes fatores de manejo, que podem ser manipulados para melhorar o uso de recursos e a eficiência da prática do consórcio em hortaliças (Bezerra Neto et al., 2003).

Face ao exposto, objetivou-se com este trabalho avaliar a viabilidade agronômica e econômica do consórcio taro e pepino, com três arranjos de plantas.

\section{MATERIAL E MÉTODOS}

O trabalho foi desenvolvido a campo, na horta da Universidade Federal de Viçosa (UFV), em Viçosa - MG, de 01/ 10/2012 a 24/06/2013. O município de Viçosa está a 650 m de altitude, $20^{\circ} 45^{\prime} 47^{\prime \prime} \mathrm{LS}$ e 4249’13" O. De acordo com a classificação de Köppen, apresenta clima tipo "Cwa” (clima subtropical úmido), com médias anuais de $1.341 \mathrm{~mm}$ de precipitação e temperaturas máxima e mínima de $21,6^{\circ} \mathrm{C}$ e de $14{ }^{\circ} \mathrm{C}$, respectivamente (INMET, 2017).

$\mathrm{O}$ solo da área experimental foi classificado como Argissolo Vermelho-Amarelo Cambissólico, textura Argilosa (Embrapa, 2006), cuja amostragem, na profundidade de $0-20 \mathrm{~cm}$, revelou os seguintes resultados: $\mathrm{pH}_{\text {(água) }}=5,6$ ; $\mathrm{P}=44,5$ e $\mathrm{K}=95 \mathrm{mg} \mathrm{dm}^{-3} ; \mathrm{Ca}^{2+}=3,2 ; \mathrm{Mg}^{2+}=0,8 \mathrm{eAl}^{3+}=$ $0,0 \mathrm{cmol}_{\mathrm{c}} \mathrm{dm}^{-3} ; \mathrm{B}=0,1 ; \mathrm{Fe}=76,4 ; \mathrm{Mn}=54,7 ; \mathrm{Zn}=22,8 \mathrm{e} \mathrm{Cu}$ $=2,1 \mathrm{mg} \mathrm{dm}^{-3} ; \mathrm{M} . \mathrm{O}=2,2 \mathrm{dag} \mathrm{kg}^{-1} ;(\mathrm{H}+\mathrm{Al})=3,30 ; \mathrm{SB}=4,24$ $; \mathrm{CTC}_{(\mathrm{t})}=4,24 \mathrm{e} \mathrm{CTC}_{(\mathrm{T})}=7,54 \mathrm{cmol}_{\mathrm{c}} \mathrm{dm}^{-3} ; \mathrm{V}=56 \%$ e P-rem $=$ $33,3 \mathrm{mg} \mathrm{L}^{-1}$. As análises foram realizadas segundo procedimento descrito por Embrapa (1999).

Os tratamentos, em número de sete, consistiram na consorciação das culturas do taro e pepino, em associação aditiva, com os respectivos cultivos solteiros, sendo: 1 - taro solteiro; 2 - consórcio, pepino com 1 planta/ cova a cada $0,30 \mathrm{~m} ; 3$ - consórcio, pepino com 2 plantas/ cova a cada $0,60 \mathrm{~m}$, opostas entre si nas linhas; 4 - consórcio, pepino com 2 plantas/cova a cada $0,60 \mathrm{~m}$, opostas alternadas entre si nas linhas; 5 - pepino solteiro, com 1 planta/cova a cada $0,30 \mathrm{~m} ; 6$ - pepino solteiro, com 2 plantas/cova a cada $0,60 \mathrm{~m}$, opostas entre si nas linhas; 7 - pepino solteiro, com 2 plantas/cova a cada $0,60 \mathrm{~m}$, opostas alternadas entre si nas linhas. Foram utilizados três tratamentos com pepino solteiro para avaliar o desempenho quando comparado com o sistema de consórcio nas diversas formas de distribuição das plantas utilizadas, ou seja, avaliar a eficiência dos consórcios. As parcelas foram constituídas de quatro linhas de 3,0 m de comprimento, espaçadas de 1,0 m, 
com dez plantas de taro e de pepino por linha, totalizando 40 plantas de cada espécie por parcela. Considerou-se como área útil a das 12 plantas das duas fileiras centrais. Utilizou-se o delineamento experimental em blocos casualizados, com quatro repetições.

O plantio do taro foi realizado em sulcos abertos à profundidade de $0,12 \mathrm{~m}$, em 01/10/2012. Foram utilizados rizomas filho de taro 'Japonês' (BGH 5925), com massa média de $60 \mathrm{~g}$, obtidos do Banco de Germoplasma de Hortaliças da UFV. O pepino verde comprido 'Híbrido Alladin F1' foi semeado em 01/10/2012, em bandejas de 128 células preenchidas com substrato comercial. O transplante das mudas, com cerca de quatro folhas definitivas, foi realizado, nos tratamentos consorciados, na mesma linha do cultivo do taro, no dia 15 de outubro de 2012.

A fertilização do solo para as culturas foi realizada com base nas análises química e física do solo e nas recomendações para as culturas, conforme Ribeiro et al. (1999). Portanto, os tratamentos em consórcio receberam as adubações recomendadas para ambas as culturas, conforme Cecílio Filho et al. (2008).

As plantas de pepino foram tutoradas individualmente, na vertical, com auxílio de fitilhos amarrados ao arame na parte inferior e em varas de bambu na parte superior, sendo retiradas todas as brotações laterais das plantas.

Durante o experimento foram realizadas três capinas com enxada. Na ausência de chuvas, até o final da colheita do pepino, foram realizadas duas irrigações semanais, com mangueiras. Após a retirada do pepino, as irrigações foram realizadas semanalmente, por aspersão, até 30 dias antes da colheita do taro. Durante o cultivo do pepino, foram necessárias aplicações de defensivos recomendados para o controle de pragas e doenças da cultura (Deltametrina, Acetamiprido, Tiofanato metílico + Clorotalonil, Oxicloreto de cobre, Tebuconazol, Azoxistrobina e Piraclostrobina). A aplicação dos produtos foi direcionada apenas às plantas de pepino.

A colheita do pepino foi parcelada, realizada duas vezes por semana, iniciando-se por volta dos 40 dias após plantio (DAP) e durando cerca de 30 dias. Em cada colheita, os frutos foram selecionados, medidos, contados e pesados. Na seleção, foram considerados impróprios para comercialização os frutos mal formados e com perfurações provocadas por insetos. Ao final da colheita, as plantas foram cortadas rente ao solo e secadas em estufa com circulação forçada de ar, a $65^{\circ} \mathrm{C}$.

Na cultura do taro, aos 60, 90, 120, 150 e 180 DAP, foram avaliados, por meio do fluorômetro modulado de pulso portátil de clorofila, os seguintes parâmetros de fluorescência: Fv/Fm (Estimativa da eficiência quântica máxima da atividade fotoquímica do PS II); Y(II) (Rendimento quântico efetivo do PS II); ETR (Taxa de transporte de elétrons); Y(NPQ) (Rendimento quântico de dissipação regulada) e Y(NO) (Rendimento quântico de dissipação não regulada). As medições foram realizadas na segunda folha completamente expandida, a partir do ápice, de duas plantas das fileiras úteis, sendo utilizada a média para representar o valor da parcela.

Aos 251 DAP do taro, as plantas da parcela útil foram colhidas e avaliadas quanto à produção de rizomas (massa de matéria fresca e número). Os rizomas-mãe foram separados e os rizomas filhos classificados com base no diâmetro transversal, de acordo com Puiatti et al. (2000), nas classes filho grande (FG), médio (FM), pequeno (FP) e refugo (REF). A produtividade total consistiu em somatório das produtividades de rizomas mãe e de todas as classes de rizomas filho. Consideraram-se comerciáveis o somatório das classes FG + FM + FP.

A eficiência dos sistemas consorciados foi medida pelos seguintes indicadores agroeconômicos: índice de uso eficiente da terra (UETm); renda bruta (RB); renda líquida (RL); vantagem monetária (VM); vantagem monetária corrigida (VMc); taxa de retorno (TR) e índice de lucratividade (IL). O índice UETm é dado pela expressão: $\mathrm{UETm}=\left(\mathrm{I}_{\text {taro }} / \mathrm{S}_{\text {taro }}\right)+\left(\mathrm{I}_{\text {pepino }} / \mathrm{S}_{\text {pepino }}\right)$, em que I e S representam as produtividades dos sistemas consorciado e solteiro de cada cultura, respectivamente.

A renda bruta (RB) foi obtida multiplicando-se a produtividade da(s) cultura(s) em cada tratamento pelo valor do produto pago ao produtor no CEASA-MG de Contagem, (CEASA-MG, 2013). Para o pepino, foram considerados os preços correspondentes a cada dia de colheita, enquanto, para o taro foram utilizados os preços do produto no dia da colheita do experimento.

A renda líquida $(\mathrm{RL})$ foi calculada, subtraindo-se da renda bruta o custo operacional total (COT), calculado para cada tratamento, levando-se em conta os coeficientes de insumos e os serviços utilizados em um hectare (ha) de taro (Puiatti, 2002) e de pepino (CEASA-MS, 2008). Os custos com insumos, serviços, embalagens e transporte foram obtidos na região de Viçosa, de outubro de 2012 a junho de 2013. A vantagem monetária e a vantagem monetária corrigida foram obtidas pelas seguintes expressões: $\mathrm{VM}=\mathrm{RB}$ x $(\mathrm{UET}-1) / \mathrm{UET}$ e VMc $=$ RL x $($ UET-1)/UET. A taxa de retorno (TR) por real investido em cada tratamento foi obtida por meio da relação entre a renda bruta $(\mathrm{RB})$ e o custo de produção (COT) de cada tratamento. $\mathrm{O}$ índice de lucratividade (IL) foi obtido da relação entre a RL e RB e expresso em percentagem, conforme Cecílio Filho et al. (2008)

Os dados obtidos foram submetidos à análise de variância. As médias dos tratamentos foram comparadas entre si pelo teste de Tukey e comparadas com as do controle pelo teste de Dunnett; os contrastes de interesse foram comparados pelo teste $\mathrm{F}$, todos a $5 \%$ de probabilidade, usando-se o programa SAEG 9.1 (SAEG, 2007). 


\section{RESULTADOS E DISCUSSÃO}

\section{Produção do pepino}

Plantas de pepino consorciadas no arranjo de 2 plantas por cova, opostas entre si (contraste Y2) e opostas alternadas entre si (contraste Y3) apresentaram maior índice de precocidade de colheita, comprimento de frutos e maior produção total de frutos, em comparação com os respectivos cultivos solteiros. $\mathrm{O}$ arranjo de duas plantas por cova, opostas entre si (contraste Y2) também apresentou maior diâmetro de frutos, maior produtividade de frutos comerciais e menor massa de matéria seca de plantas, em comparação com o cultivo solteiro (Tabela1).

A produtividade de frutos comerciais de pepino variou de 54,43 tha $^{-1}$ (monocultivo de 2 plantas por cova, opostas entre si nas linhas), a 69,81 t ha-1 (consórcio 1 planta por cova). Com esse mesmo híbrido (Aladin F1), Sediyama et al. (2014) obtiveram produtividade máxima de 40,16 t.ha ${ }^{-1}$ na Zona da Mata Mineira, utilizando apenas o esterco bovino curtido como fonte de nutrientes.

O consórcio de taro e pepino, nos arranjos avaliados, não afetou a produtividade comercial de frutos de pepino, sendo que, nos arranjos com duas plantas por cova, as produtividades de frutos comerciais foram até superiores à do monocultivo. Por apresentar crescimento mais rápido que o taro, as plantas de pepino, no sistema de consórcio, podem ter aproveitado parte dos nutrientes que foram destinados à cultura do taro. $\mathrm{O}$ fato de as plantas de pepino nos consórcios apresentarem menor índice de precocidade é uma evidência para isso.

São poucos os trabalhos que tratam do consórcio do pepino com outras hortaliças. Rezende et al. (2010) avaliaram os consórcios de alface crespa e pepino, em função da população do pepino e da época de cultivo e verificaram que a produtividade e a classificação dos frutos de pepino não foram influenciadas pela presença da alface. Quando avaliaram a viabilidade da consorciação de pimentão com repolho, rúcula, alface e rabanete, Rezende et al. (2006) observaram que as plantas de pimentão apresentaram rápido estabelecimento de dossel fotossintético, acima das demais culturas em associação e não tiveram a produtividade influenciada pelo sistema de cultivo.

\section{Produção do taro}

Exceto rizomas mãe e filho médio, todos os arranjos dos consórcios adotados afetaram a produção do taro, em comparação com os do controle (taro solteiro). Comparados entre si, os arranjos tiveram efeito semelhante sobre a produção do taro, sendo que apenas o arranjo duas plantas de pepino por cova, alternadas, espaçadas de $60 \mathrm{~cm}$, produziu mais FP e NFCP que o arranjo com duas plantas de pepino por cova, opostas, espaçadas de $60 \mathrm{~cm}$ (Tabela 2).

A produtividade de rizomas comerciais variou de 18,83 t ha ${ }^{-1}$ (consórcio 2 plantas por cova, opostas entre si nas linhas) a 29,17 t ha-1 (taro solteiro). Nas condições de Viçosa-MG, também com o taro 'Japonês', Gondim et al. (2007) obtiveram produtividade de 41,69 $\mathrm{t} \mathrm{ha}^{-1} \mathrm{com}$ o taro cultivado a pleno sol, sendo que variou de 22,3 a $35,0 \mathrm{t}$ ha $^{-1}$ quando cultivado sob sombreamento artificial de 50, 30 e $18 \%$, durante determinadas fases do ciclo cultural. A intensidade de $18 \%$ de sombra, durante todo o ciclo, ou nos períodos inicial (0-3 meses) e intermediário (3-6 meses), foi a que menos afetou o desenvolvimento das plantas e a produção de biomassa de rizomas comerciáveis.

Tabela 1: Valores médios observados nos contrastes entre tratamentos de cultivos consorciado e solteiro de pepino (Y1, Y2, Y3), nos três arranjos de plantas utilizados, das características número de frutos total por planta (NFTP), número de frutos comerciais por planta (NFCP), índice de frutos não comerciais por planta (IFNCP), índice de precocidade da colheita (IPC), comprimento dos frutos (CF), diâmetro dos frutos (DF), produção total de frutos (PTF), produção de frutos comercias (PFC) e massa de matéria seca de plantas (MSP), após a colheita de todos os frutos

\begin{tabular}{|c|c|c|c|c|c|c|c|c|c|c|}
\hline \multirow{2}{*}{ Contrastes } & \multirow{2}{*}{ Tratamentos } & NFTP & NFCP & IFNCP & IPC & CF & DF & PTF & PFC & MSP \\
\hline & & \multicolumn{2}{|c|}{ unid } & \multicolumn{2}{|c|}{$\%$} & \multicolumn{2}{|c|}{$\mathrm{cm}$} & \multicolumn{3}{|c|}{ t/ha ${ }^{-1}$} \\
\hline & 2 & 10,79 & 9,59 & 11,11 & 22,17 & 17,64 & 4,39 & 73,41 & 69,81 & 1,22 \\
\hline & 5 & 10,77 & 9,11 & 15,58 & 20,82 & 17,60 & 4,46 & 71,41 & 66,91 & 1,10 \\
\hline \multirow[t]{3}{*}{$\mathrm{Y} 1$} & & $0,02^{\mathrm{ns}}$ & $0,48^{\mathrm{ns}}$ & $-4,47^{\mathrm{ns}}$ & $1,35^{\mathrm{ns}}$ & $0,04^{\mathrm{ns}}$ & $-0,07^{\mathrm{ns}}$ & $2,00^{\mathrm{ns}}$ & $2,90^{\mathrm{ns}}$ & $0,12^{\mathrm{ns}}$ \\
\hline & 3 & 9,61 & 8,54 & 11,06 & 20,82 & 17,69 & 4,50 & 64,72 & 61,87 & 0,97 \\
\hline & 6 & 9,13 & 8,25 & 9,56 & 9,86 & 16,22 & 4,26 & 57,17 & 54,43 & 1,23 \\
\hline \multirow[t]{3}{*}{ Y2 } & & $0,48^{\mathrm{ns}}$ & $0,29^{\mathrm{ns}}$ & $1,5^{\mathrm{ns}}$ & $10,96^{* *}$ & $1,47 * *$ & $0,24 * *$ & $7,55^{*}$ & $7,44^{*}$ & $-0,26 * *$ \\
\hline & 4 & 10,00 & 8,83 & 11,53 & 21,30 & 17,35 & 4,45 & 67,31 & 63,95 & 1,12 \\
\hline & 7 & 9,75 & 8,67 & 11,04 & 13,19 & 16,42 & 4,35 & 61,15 & 58,46 & 1,27 \\
\hline $\mathrm{Y} 3$ & & $0,25^{\mathrm{ns}}$ & $0,16^{\mathrm{ns}}$ & $0,49^{\text {ns }}$ & 8,11 ** & $0,93 * *$ & $0,10^{\text {ns }}$ & $6,16^{*}$ & $5,49^{\text {ns }}$ & $-0,15^{\mathrm{ns}}$ \\
\hline
\end{tabular}

Tratamentos: 2 = Consórcio $/ 1 \mathrm{pl} / \mathrm{cova} / 30 \mathrm{~cm} ; 3=$ Consórcio $/ 2 \mathrm{pl} / \mathrm{cova} / 60 \mathrm{~cm} /$ opostas entre si nas linhas; 4 = Consórcio/2pl $/ \mathrm{cova} / 60 \mathrm{~cm} /$ opostas alternadas entre si nas linhas; 5,6 e $7=$ monocultivos do pepino relativos aos arranjos dos tratamentos, 2,3 e 4 , respectivamente. $\mathrm{ns}=$ não significativo; $*$ = significativo pelo teste $\mathrm{F}$ a $5 \%$ de probabilidade; $* *=$ significativo pelo teste $\mathrm{F}$ a $1 \%$ de probabilidade. 
Neste experimento, a cultura do pepino em consórcio permaneceu por 71 dias, o que estaria no período inicial de cultivo do taro; todavia, mesmo assim, causou redução média de cerca de $29 \%$ na produção de rizomas comerciais.

O taro consorciado com outras olerícolas tem apresentado resultados diversos. Maior produção comercial do taro 'Chinês' em cultivo solteiro, em comparação com a do consorciado com chicória, foi observada por Heredia Zárate et al. (2007b). Entretanto, quando o taro 'Chinês' foi consorciado com a alface 'Quatro Estações', apresentou maior produção comercial (Heredia Zárate et al., 2006). No consórcio taro e milho doce 'Cristal', plantado 40 dias após o taro, a produção de rizomas foi afetada em relação à da monocultura do taro (Puiatti et al., 2000). Em cultivo intercalar com a Crotalaria juncea, o taro apresentou menor produtividade de rizomas filho quando a leguminosa não foi podada durante o desenvolvimento do consórcio (Oliveira et al., 2007). Em outro trabalho, Oliveira et al. (2004) não verificaram queda da produtividade do taro, mesmo quando a leguminosa permaneceu em associação permanente.

Por causa do crescimento indeterminado e do cultivo tutorado, as plantas de pepino promoveram sombreamento das plantas de taro durante aproximadamente dois meses. Gondim et al. (2007) verificaram que o sombreamento artificial de $30 \%$ na fase inicial (0-3 meses) afetou a produtividade total de rizomas, comerciais, número de rizomas comerciais, filhos médio e refugo e o número de rizomas comerciais por planta, e o de $50 \%$ de sombreamento afetou também a produtividade de rizomas filho grande.

Condições de acentuada restrição de luz favorecem o investimento, por parte das plantas, no crescimento da parte aérea em detrimento da formação dos órgãos de reserva, levando à redução da produção de rizomas (Gondim et al., 2007; Oliveira et al., 2011).

\section{Fluorescência da clorofila}

No trabalho com consórcios, por causa do efeito do sombreamento, é fundamental avaliar o desempenho fotossintético das plantas. Entre as técnicas utilizadas, as medições de fluorescência da clorofila têm merecido destaque. O rendimento quântico do fotossistema II (PS II), obtido a partir da fluorescência da clorofila a, pode revelar o nível energético de excitação dos pigmentos que dirigem a fotossíntese; a razão $\mathrm{Fv} / \mathrm{Fm}$ pode ser um indicativo de eficiência no uso da radiação pela fotoquímica, e, consequentemente, a assimilação de carbono. Assim, as medições de fluorescência da clorofila são usadas com eficiência na avaliação do desempenho fotossintético.

Nas avaliações relativas à fluorescência da clorofila nas plantas de taro, não foi verificada diferença significativa entre os tratamentos para o parâmetro Fv/Fm. Nas avaliações realizadas até aos 150 DAP do taro, para todos os tratamentos, os valores médios observados foram superiores a 0,75 . Porém, na avaliação realizada aos 180 DAP, para todos os tratamentos, os valores foram inferiores a 0,75 (Tabela 3). A razão Fv/Fm é uma estimativa da eficiência quântica máxima da atividade fotoquímica do PSII, quando todos seus centros de reação estão abertos (Baker \& Rosenqvst, 2004). A queda da atividade fotoquímica do PSII na última avaliação é explicada pela razão de que, no taro, a partir dos 165 DAP, ocorre decréscimo do acúmulo de massa de matéria fresca na parte aérea, em razão do processo de senescência foliar (Puiatti et al., 1992). Segundo BolhárNordenkampf \& Ôquist (1993), plantas que não estão sob estresse apresentam relação Fv/Fm entre 0,85 e 0,75. Valores menores que 0,75 seriam indicativos de condições inibitórias do PSII.

O rendimento quântico efetivo do PSII, o Y (II) ou $\triangle \mathrm{F} / \mathrm{F}$ 'm, está relacionado com a estimativa da disponibi-

Tabela 2: Rendimentos, em massa de matéria fresca, de rizomas mãe (RM), total (RT), comercial (RC), filho grande (FG), filho médio (FM), filho pequeno (FP), filho refugo (REF) e número de rizomas filho comerciais por planta (NFCP), em plantas de taro em monocultivo (cultivo solteiro) e em consórcios com pepino

\begin{tabular}{|c|c|c|c|c|c|c|c|c|}
\hline \multirow{2}{*}{ Tratamento } & $\mathbf{R M}$ & RT & $\mathbf{R C}$ & FG & FM & FP & REF & \multirow{2}{*}{$\frac{\text { NFCP }}{\text { ud }}$} \\
\hline & \multicolumn{7}{|c|}{ t ha $^{-1}$} & \\
\hline 1 - Taro solteiro & $7,46 a$ & $43,04 \mathrm{a}$ & $29,17 \mathrm{a}$ & $10,59 \mathrm{a}$ & $8,58 \mathrm{a}$ & $10,00 \mathrm{a}$ & $6,41 \mathrm{a}$ & $11,31 \mathrm{a}$ \\
\hline 2 - Consórcio - 1 pl/cova & $6,55 \mathrm{a}$ & $30,55 b^{*}$ & $20,84 b^{*}$ & $6,99 a b^{*}$ & $7,29 \mathrm{a}$ & $6,56 \mathrm{bc} *$ & $3,16 b^{*}$ & $8,39 \mathrm{bc}^{*}$ \\
\hline $\begin{array}{l}3 \text { - Consórcio - } 2 \mathrm{pl} / \text { cova - opostas } \\
\text { entre si nas linhas }\end{array}$ & $6,49 a$ & $29,10 b^{*}$ & $18,83 b^{*}$ & $6,80 \mathrm{ab} *$ & $5,75 \mathrm{a}$ & $6,28 c^{*}$ & $3,78 b^{*}$ & $7,60 c^{*}$ \\
\hline $\begin{array}{l}4 \text { - Consórcio - } 2 \text { pl/cova - opostas alternadas } \\
\text { entre si nas linhas }\end{array}$ & $6,98 \mathrm{a}$ & $32,90 b^{*}$ & $22,54 b^{*}$ & $6,14 b^{*}$ & $7,95 \mathrm{a}$ & $8,45 \mathrm{ab}$ & $3,38 b^{*}$ & $10,00 \mathrm{ab}$ \\
\hline CV\% & 13,84 & 11,50 & 14,66 & 34,49 & 34,45 & 15,53 & 28,88 & 13,71 \\
\hline Média & 6,87 & 33,90 & 22,84 & 7,63 & 7,39 & 7,82 & 8,83 & 9,33 \\
\hline
\end{tabular}

Médias, nas colunas, seguidas de pelo menos uma mesma letra, não diferem entre si pelo teste de Tukey a 5\% de probabilidade; médias, nas colunas, seguidas de asterisco $(*)$, diferem do controle a $5 \%$ de probabilidade. 
lidade de dissipação de energia para o metabolismo do carbono e o ETR, a taxa de transporte de elétrons. Neste trabalho, na avaliação realizada aos 60 DAP, os tratamentos de consórcio apresentaram menores valores de Y(II) e de ETR, que os do controle (Tabela 3). Aos 60 DAP, as plantas de taro encontravam-se sob sombreamento proporcionado pelas plantas de pepino. Na avaliação realizada aos 120 DAP, os valores observados, no tratamento em que foi cultivada uma planta de pepino a cada $30 \mathrm{~cm}$, foram inferiores aos observados no tratamento em que as plantas de pepino foram cultivadas a cada $60 \mathrm{~cm}$, opostas entre si nas linhas. Esse comportamento pode ser reflexo do período em que as plantas de taro encontravam-se sombreadas, uma vez que no tratamento em que as plantas de pepino foram cultivadas a cada $30 \mathrm{~cm}$ pode ter ocorrido maior sombreamento, afetando a dissipação de energia para o metabolismo do carbono, mesmo após a retirada de plantas de pepino, por causa do estresse provocado pelo sombreamento. Para as demais épocas, não houve diferença significativa, quando em comparação com o cultivo do taro solteiro.

Aos 60 DAP, os tratamentos de consórcio apresentaram valores superiores ao do controle, quanto ao rendimento quântico de dissipação regulada Y(NPQ) (Tabela 3). Segundo Li et al. (2010), o aumento do NPQ sugeriu o mecanismo para minimizar o estresse com a dissipação da energia em processos não fotoquímicos na forma de calor. Esta é uma das estratégias de proteção do aparato fotossintético contra o excesso de energia fotossintetizante. Segundo Havaux \& Niyogi (1999), em plantas sob condições de estresse, a menor eficiência fotossintética pode ser causada pela menor dissipação de energia, por meio do transporte de elétrons, ocasionando declínio da eficiência quântica potencial do PSII, indicada pelo menor

Tabela 3: Parâmetros de fluorescência da clorofila: estimativa da eficiência quântica máxima da atividade fotoquímica do PSII (Fv/ Fm); rendimento quântico do PSII Y(II);taxa de transporte de elétrons (ETR); rendimento quântico de dissipação regulada Y(NPQ) e rendimento quântico de dissipação não regulada $\mathrm{Y}(\mathrm{NO})$, medidos aos 60, 90, 120, 150 e 180 dias após o plantio do taro (DAP), em folhas de taro cultivado solteiro (controle) e em consórcios com o pepino, estabelecido com 1 ou 2 plantas por cova, opostas entre si e opostas alternadas entre si nas linhas

\begin{tabular}{|c|c|c|c|c|c|}
\hline \multirow{2}{*}{ Variável } & \multicolumn{5}{|c|}{ Tratamentos* } \\
\hline & DAP & 1 & 2 & 3 & 4 \\
\hline \multirow{5}{*}{$\mathrm{Fv} / \mathrm{Fm}$} & 60 & $0,79 a$ & $0,80 \mathrm{a}$ & $0,76 a$ & $0,78 \mathrm{a}$ \\
\hline & 90 & $0,78 \mathrm{a}$ & $0,78 \mathrm{a}$ & $0,77 \mathrm{a}$ & $0,79 \mathrm{a}$ \\
\hline & 120 & $0,78 \mathrm{a}$ & $0,79 a$ & $0,77 \mathrm{a}$ & $0,78 \mathrm{a}$ \\
\hline & 150 & $0,76 a$ & $0,76 a$ & $0,78 \mathrm{a}$ & $0,77 \mathrm{a}$ \\
\hline & 180 & $0,68 \mathrm{a}$ & $0,68 \mathrm{a}$ & $0,68 \mathrm{a}$ & $0,69 \mathrm{a}$ \\
\hline \multirow{5}{*}{ Y (II) } & 60 & $0,31 \mathrm{a}$ & $0,16 b^{*}$ & $0,15 b^{*}$ & $0,13 b^{*}$ \\
\hline & 90 & $0,23 \mathrm{a}$ & $0,21 \mathrm{a}$ & $0,19 a$ & $0,21 \mathrm{a}$ \\
\hline & 120 & $0,23 \mathrm{ab}$ & $0,18 b$ & $0,24 \mathrm{a}$ & $0,21 \mathrm{ab}$ \\
\hline & 150 & $0,23 \mathrm{a}$ & $0,27 \mathrm{a}$ & $0,27 \mathrm{a}$ & $0,28 \mathrm{a}$ \\
\hline & 180 & $0,13 \mathrm{a}$ & $0,16 \mathrm{a}$ & $0,16 a$ & $0,17 \mathrm{a}$ \\
\hline \multirow{5}{*}{ ETR } & 60 & $183,99 a$ & $93,65 b^{*}$ & $90,36 b^{*}$ & $77,58 \mathrm{~b} *$ \\
\hline & 90 & $137,74 \mathrm{a}$ & $127,07 \mathrm{a}$ & $114,62 \mathrm{a}$ & $126,47 \mathrm{a}$ \\
\hline & 120 & $135,11 \mathrm{ab}$ & $103,72 b$ & $138,97 \mathrm{a}$ & $125,63 \mathrm{ab}$ \\
\hline & 150 & $134,71 \mathrm{a}$ & $157,75 \mathrm{a}$ & $159,10 \mathrm{a}$ & $163,77 \mathrm{a}$ \\
\hline & 180 & $79,24 \mathrm{a}$ & $97,31 \mathrm{a}$ & $94,57 \mathrm{a}$ & $101,82 \mathrm{a}$ \\
\hline \multirow{5}{*}{ Y (NPQ) } & 60 & $0,38 b$ & $0,48 a^{*}$ & $0,51 \mathrm{a}^{*}$ & $0,48 a^{*}$ \\
\hline & 90 & $0,43 \mathrm{a}$ & $0,45 \mathrm{a}$ & $0,45 \mathrm{a}$ & $0,45 \mathrm{a}$ \\
\hline & 120 & $0,50 \mathrm{a}$ & $0,50 \mathrm{a}$ & $0,48 \mathrm{a}$ & $0,48 \mathrm{a}$ \\
\hline & 150 & $0,46 \mathrm{a}$ & $0,43 \mathrm{a}$ & $0,43 \mathrm{a}$ & $0,43 a$ \\
\hline & 180 & $0,47 \mathrm{a}$ & $0,48 \mathrm{a}$ & $0,46 \mathrm{a}$ & $0,47 \mathrm{a}$ \\
\hline \multirow{5}{*}{$\mathrm{Y}(\mathrm{NO})$} & 60 & $0,31 \mathrm{a}$ & $0,36 \mathrm{a}$ & $0,33 \mathrm{a}$ & $0,39 \mathrm{a}$ \\
\hline & 90 & $0,34 \mathrm{a}$ & $0,33 \mathrm{a}$ & $0,36 \mathrm{a}$ & $0,34 \mathrm{a}$ \\
\hline & 120 & $0,29 a$ & $0,33 \mathrm{a}$ & $0,28 \mathrm{a}$ & $0,30 \mathrm{a}$ \\
\hline & 150 & $0,31 \mathrm{a}$ & $0,30 \mathrm{a}$ & $0,30 \mathrm{a}$ & $0,30 \mathrm{a}$ \\
\hline & 180 & $0,40 \mathrm{a}$ & $0,36 \mathrm{a}$ & $0,38 \mathrm{a}$ & $0,35 \mathrm{a}$ \\
\hline
\end{tabular}

Médias nas linhas, seguidas de pelo menos uma mesma letra, não diferem entre si pelo teste de Tukey a 5\% de probabilidade; médias, nas linhas, seguidas de asterisco (*), diferem do controle a $5 \%$ de probabilidade

*Tratamento: 1 = Controle (monocultivos do taro); 2 = Consórcio/1pl $/ \mathrm{cova} / 30 \mathrm{~cm} ; 3=$ Consórcio/2pl $/ \mathrm{cova} / 60 \mathrm{~cm} / \mathrm{opostas}$ entre si nas linhas; 4 = Consórcio/2pl/cova/60cm/opostas alternadas entre si nas linhas 
$\mathrm{F} v / \mathrm{Fm}$, e da taxa de transporte de elétrons (ETR) sendo, usualmente, associados ao aumento da extinção não fotoquímica da fluorescência, por meio da dissipação regulada, e do "pool" de zeaxantina.

Aos 60 DAP, as plantas de taro encontravam-se totalmente cobertas pelas plantas de pepino, recebendo, assim, elevado grau de sombreamento, causando estresse nas plantas e elevando os valores de Y (NPQ). Com a retirada das plantas de pepino, que ocorreu aproximadamente aos 70 DAP, todas as plantas de taro passaram a ter a mesma condição de luminosidade, não sendo verificadas diferenças entre os tratamentos.

Não houve diferença do rendimento quântico de dissipação não regulada $\mathrm{Y}(\mathrm{NO})$ entre os tratamentos, nas avaliações (Tabela 3). Apesar disso, os valores médios dos tratamentos de consórcio, por ocasião da avaliação realizada aos 60 DAP, foram superiores aos do controle, indicando maior fotodano por ocasião do elevado grau de sombreamento, proporcionado pela cultura do pepino cultivado na forma de consórcio. Esse parâmetro de fluorescência é um indicativo de fotoinibição. Huang et al. (2010) verificaram que os valores de $\mathrm{Y}(\mathrm{NO})$ correlacionaram-se negativamente com $\mathrm{Fv} / \mathrm{Fm}$, concluindo que, além de Fv/Fm, o Y(NO) é outro bom indicador de fotodano ao PSII. Portanto, efeitos negativos observados quanto aos parâmetros da fluorescência da clorofila podem explicar, em parte, os menores valores de produção de rizomas de taro, obtidos nos tratamentos em associação com pepino.

\section{Indicadores agroeconômicos}

Os consórcios apresentaram valores UETm variando de 1,76 a 1,84, sem diferirem entre si, e superiores aos dos monocultivos (Tabela 4). Isso significa que seriam neces- sários de 76 a 84\% mais de área para que as culturas em plantio solteiro produzissem o equivalente à produção dessas culturas consorciadas. Portanto, os consórcios mostraram melhor aproveitamento dos fatores ambientais disponíveis que os monocultivos.

Valores superiores de UETm também foram encontrados em cultivo consorciado de taro e alface (Heredia Zárate et al., 2005). Nos consórcios taro e cenoura e taro e alface, Heredia Zárate et al. (2006) obtiveram UETm de 6 e de $83 \%$, respectivamente. Rezende (2008), em consórcio pepino e alface, verificou o maior valor de $\operatorname{UETm}(1,68)$ correspondente ao consórcio com alface americana, com uma linha de plantas de pepino no centro do canteiro.

Resultado semelhante ao UETm foi observado para renda bruta, para a qual todos os tratamentos de consórcio apresentaram valores superiores aos do monocultivo do taro (controle). Entretanto, para renda líquida, não houve diferença entre tratamentos de consórcio e de controle. A explicação está no maior valor do COT do sistema de consórcio ( $\mathrm{R}$ \$ 42.281,80/ha), comparado com o do COT do monocultivo do taro ( $\mathrm{R} \$ 14.653,78 / \mathrm{ha})$, associado ao menor valor médio de comercialização do pepino por ocasião de sua colheita ( $\mathrm{R} \$ 12,30$ / cx 19kg), comparado com o valor de comercialização do taro por ocasião de sua colheita (R\$38,00/ cx 19kg e R \$30,00/ sc 19kg, em função da classificação pelo tamanho, maior e menor, respectivamente ).

Valores superiores de renda bruta também foram encontrados em cultivos consorciados de taro e alface, comparados com os do controle (Heredia Zárate et al., 2005). No consórcio pepino e alface, Rezende (2008) verificou que as receitas brutas e líquidas dos consórcios foram sempre superiores às obtidas em monoculturas, quando o transplante

Tabela 4: Indicadores agroeconômicos, Índice de Uso da Terra (UETm), Renda Bruta (RB), Renda Líquida (RL), Vantagem Monetária (VM), Vantagem Monetária corrigida (VMc), Taxa de Retorno (TR) e Índice de Lucratividade (IL), observados no consórcio taro e pepino e nos monocultivos

\begin{tabular}{|c|c|c|c|c|c|c|c|}
\hline \multirow{2}{*}{ Tratamento } & UETm & RB & $\mathbf{R L}$ & VM & VMc & \multirow{2}{*}{$\frac{\mathrm{TR}}{(\mathbf{R} \$ \mathbf{R} \$)}$} & \multirow{2}{*}{$\frac{\text { IL }}{(\%)}$} \\
\hline & & \multicolumn{4}{|c|}{$\left(\mathbf{R} \$ h^{-1}\right)$} & & \\
\hline 1 - Taro solteiro & $1,00 \mathrm{~b}$ & $50522,01 \mathrm{~b}$ & $35181,54 \mathrm{a}$ & 0,0 & 0,0 & $3,29 \mathrm{a}$ & $69,53 \mathrm{a}$ \\
\hline 2 - Consórcio - 1 pl/cova & $1,76 a^{*}$ & $79685,39 a^{*}$ & $36776,84 \mathrm{a}$ & 34376,83 a & $15931,14 \mathrm{a}$ & $1,85 b^{*}$ & $45,86 b^{*}$ \\
\hline $\begin{array}{l}3 \text { - Consórcio - } 2 \text { pl/covaopostas } \\
\text { entre si nas linhas }\end{array}$ & $1,78 a^{*}$ & $71602,13 \mathrm{a}^{*}$ & $30818,64 \mathrm{a}$ & $31354,38 \mathrm{a}$ & $13608,42 \mathrm{a}$ & $1,75 b^{*}$ & $42,77 b^{*}$ \\
\hline $\begin{array}{l}4 \text { - Consórcio - } 2 \mathrm{pl} / \text { cova opostas } \\
\text { alternadas entre si nas linhas }\end{array}$ & $1,84 a^{*}$ & $78175,64 a^{*}$ & $36369,20 \mathrm{a}$ & $35682,58 \mathrm{a}$ & 16756,06 a & $1,86 b^{*}$ & $46,03 b^{*}$ \\
\hline 5 - Pepino solteiro - $1 \mathrm{pl} /$ cova & $1,00 \mathrm{~b}$ & $41715,23 \mathrm{bc}^{*}$ & $11838,75 b^{*}$ & 0,0 & 0,0 & $1,39 \mathrm{c}^{*}$ & $27,84 \mathrm{c}^{*}$ \\
\hline $\begin{array}{l}6 \text { - Pepino solteiro - } 2 \mathrm{pl} / \text { cova opostas } \\
\text { entre si nas linhas }\end{array}$ & $1,00 \mathrm{~b}$ & $32234,23 \mathrm{c} *$ & $5100,68 \mathrm{~b}^{*}$ & 0,0 & 0,0 & $1,19 \mathrm{c}^{*}$ & $15,75 \mathrm{~cd}^{*}$ \\
\hline $\begin{array}{l}7 \text { - Pepino solteiro - } 2 \mathrm{pl} / \text { cova opostas } \\
\text { alternadas entre si nas linhas }\end{array}$ & $1,00 \mathrm{~b}$ & $36035,79 \mathrm{c} *$ & $8018,22 b^{*}$ & 0,0 & 0,0 & $1,28 \mathrm{c} *$ & $22,22 \mathrm{~d}^{*}$ \\
\hline $\mathrm{CV}(\%)$ & 8,98 & 10,34 & 20,50 & 33,76 & 46,84 & 6,36 & 9,30 \\
\hline
\end{tabular}

Médias, nas colunas, seguidas de pelo menos uma mesma letra, não diferem entre si pelo teste de Tukey a 5\% de probabilidade; médias, nas colunas, seguidas de asterisco $(*)$, diferem do controle a $5 \%$ de probabilidade. 
de pepino e alface ocorreram no mesmo dia, independentemente da densidade populacional do pepino.

Em relação à vantagem monetária e à vantagem monetária corrigida não houve diferença entre os tratamentos de consórcio; contudo, para a taxa de retorno e o índice de lucratividade, os valores dos tratamentos de consórcio mostraram-se inferiores ao controle, provavelmente pelo baixo valor de mercado apresentado pelo pepino durante o período da colheita.

Neste trabalho, a superioridade agronômica verificada por meio da UETm não resultou em vantagem econômica. Heredia Zárate et al. (2007a) também verificaram que a maior UETm não induziu à maior renda líquida no consórcio do taro macaquinho com a alface. Rezende et al. (2005), avaliando consórcios de alface e tomate, verificaram que, embora os UETm apresentassem elevada superioridade (até $79 \%$ dos cultivos consorciados sobre as monoculturas), não foi constatada a mesma amplitude para o lucro. Os autores atribuíram o baixo valor da alface recebido na época da colheita, como não contribuindo substancialmente para a receita auferida no consórcio, representando apenas $7 \%$ na sua composição.

De acordo com Harder et al. (2005), o aumento da produtividade por unidade de área é uma das razões mais importantes para se cultivar duas ou mais culturas no sistema de consorciação. Esse sistema permite melhor aproveitamento da terra e de outros recursos disponíveis, resultando, na maioria das vezes, em maior rendimento econômico. Todavia, para Beltrão et al. (1984), o UETm fornece apenas a dimensão física do sistema, sem considerar o valor de cada componente do sistema consorciado.

Deve-se ressaltar que as hortaliças apresentam variações no preço e no custo de produção ao longo do ano. Embora as produtividades das culturas sejam dependentes da época de cultivo, a influência da sazonalidade de preços das hortaliças envolvidas no consórcio, ao longo do ano, permite obter informações sobre como a rentabilidade econômica de determinado cultivo pode sofrer variação.

Cabe ressaltar que, embora a renda líquida, a taxa de retorno e o índice de lucratividade dos consórcios não tenha sido superior, a entrada de capital de forma antecipada já aos 71 dias do plantio é muito importante para o pequeno produtor, pois o cultivo solteiro do taro somente traria receita após nove meses do plantio.

$\mathrm{Na}$ avaliação dos indicadores agroeconômicos, apenas as produtividades obtidas das culturas envolvidas no consórcio foram consideradas. A produtividade é a variável a que é dada maior importância no momento de definir um sistema de cultivo; entretanto, cabe destacar a importância da tecnologia da consorciação, em relação à do monocultivo, quanto à sustentabilidade do ambiente (Innis, 1997).

\section{CONCLUSÕES}

Apesar de o consórcio com o pepino ter afetado a produtividade do taro, os três arranjos avaliados foram agronômica e economicamente viáveis, além da entrada de recursos antecipada com a colheita do pepino.

\section{AGRADECIMENTOS}

Os autores agradecem ao CNPq e à FAPEMIG, pelo suporte financeiro para realização deste trabalho.

\section{REFERÊNCIAS}

Baker NR \& Rosenqust E (2004) Aplication of chlorophyll fluorescence can improve crop production strategies: an examination of future possibilities. Journal of Experimental Botany, 55:1607-1621.

Barros Júnior AP, Bezerra Neto F, Negreiros MZ, Oliveira EQ, Silveira LM \& Câmara MJT (2005) Desempenho agronômico do bicultivo da alface em sistemas consorciados com cenoura em faixa sob diferentes densidades populacionais. Horticultura Brasileira, 23:712-717.

Beltrão NEM, Nobrega LB, Azevedo DMP \& Vieira DJ (1984) Comparação entre indicadores agroeconômicos de avaliação de agroecossistemas consorciados e solteiros envolvendo algodão "upland" e feijão "caupi". Campina Grande, Embrapa-CNPA. 21p. (Boletim técnico, 15).

Bezerra Neto F, Andrade FV, Negreiros MZ \& Santos Júnior JS (2003) Desempenho agroeconômico do consórcio cenoura $\mathrm{x}$ alface lisa em dois sistemas de cultivo. Horticultura Brasileira, 21:635-641

Bolhár-Nordenkampf \& HR Öquist G (1993) Chlorophyll fluorescence as a tool in photosynthesis research. In: Hall DO, Scurlock JMO, Bolhár-Nordenkampf HR, Leegood RC \& Long SP (Eds.) Photosynthesis and production in changing environment: a field and laboratory manual. London, Chapman e Hall. p. 193-206.

CEASA - Centrais de abastecimento de Mato Grosso do Sul (2008) Custos de Produção. Seminário de Horticultura CEASA_MS. Campo Grande, CEASA. 123p.

CEASA - Centrais de abastecimento de Minas Gerais (2013) Lista Completa de Preços dos Produtos nas Unidades da Ceasa Minas. Disponível em: <http://minas.ceasa.mg.gov.br/detec/ oferta_preco/>. Acessado em 07 de dezembro de 2013.

Cecílio Filho AB, Costa CC, Rezende BLA \& Leeuwen R (2008) Viabilidade produtiva e econômica do consórcio entre chicória e rúcula em função da época de plantio. Horticultura Brasileira, 26:316-320.

CONAB (2015) Agricultura familiar. Disponível em: <http:// www.conab.gov.br/conteudos.php?a=1125>. Acessado em: 19 de janeiro de 2015.

Embrapa - Empresa Brasileira de Pesquisa Agropecuária (1999) Manual de análises químicas de solos, plantas e fertilizantes. Brasília, Embrapa Comunicação para Transferência de Tecnologia. 370p.

Embrapa - Empresa Brasileira de Pesquisa Agropecuária (2006) Sistema brasileiro de classificação de solos. $2^{a}$ ed. Rio de Janeiro, Embrapa. 306p

Gondim ARO, Puiatti M, Cecon PR \& Finger FL (2007) Crescimento, partição de fotoassimilados e produção de rizomas em taro cultivado sob sombreamento artificial. Horticultura Brasileira, 25:418-428. 
Harder WC, Heredia Zarate NA \& Vieira MC (2005) Produção e renda bruta de rúcula (Eruca Sativa Mill.) 'cultivada' e de almeirão (cichorium intybus L.) 'amarelo' em cultivo solteiro e consorciado. Ciência e Agrotecnologia, 29:775-785.

Havaux M \& Niyogi KK (1999) The violaxanthin cycle protects plants from photooxidative damage by more than one mecanism. Proceedings of the National Academy of Sciences of the United States of America, 96:8762-8767.

Heredia Zárate NA, Vieira MC, Oliveira ACP \& Lima AA (2005) Produção e renda bruta de dois cultivares de taro, em cultivo solteiro e consorciado com alface. Semina: Ciências Agrárias, 26:283-290.

Heredia Zárate NA, Vieira MC, Giuliani AR, Helmich M, Chiquito EG \& Amadori AH (2006) Taro 'Chinês' em cultivo solteiro e consorciado com cenoura 'Brasília' e alface 'Quatro Estações'. Horticultura Brasileira, 24:324-328.

Heredia Zárate NA, Vieira MC, Giuliani AR, Helmich M, Pontim BCA, Pezzoni Filho JC (2007a) Produção e renda de taro Macaquinho, solteiro e consorciado com alface 'Salad Bowl', em solo com cobertura de cama-de-frango semidecomposta. Semina: Ciências Agrárias, 28:563-570.

Heredia Zárate NA, Vieira MC, Graciano JD, Helmich M, Gassi RP \& Souza CM (2007b) Produção do taro chinês, em cultivo solteiro e consorciado com chicória. Ciência e Agrotecnologia, $31: 1558-1562$.

Huang W, Zhang S \& Cao K (2010) Stimulation of Cyclic Electron Flow During Recovery After Chilling - Induced Photoinhibition of PS II. Plant Cell Physiology, 51:1922-1928.

IBGE - Instituto Brasileiro de Geografia e Estatística (2006) Censo Agropecuário: Brasil, Grandes Regiões e Unidades da Federação. Rio de Janeiro, IBGE.777p.

INMET - Instituto Nacional de Meteorologia (2017) Normais Climatológicas. Disponível em: <http://www.inmet.gov.br/portal/index.php? $\mathrm{r}=$ clima/normaisclimatologicas $>$. Acessado em: 20 de novembro de 2017.

Innis DQ (1997) Intercropping and the scientific basis of the traditional agriculture. London, Intermediate. 179p.

Li G, Wab S, Zhou J, Yang Z \& Qin P (2010) Leaf chlorophyll fluorescence, hyperspectral reflectance, pigments content, malondialdehyde and proline accumulation responses of castor bean (Ricinuscommunis L.) seedlings to salt stress level. Industrial Cropsand Products, 31:13-19.

Oliveira FL, Ribeiro RLD, Silva VV, Guerra JGM \& Almeida DL (2004) Desempenho do inhame (taro) em plantio direto e no consórcio com crotalária, sob manejo orgânico. Horticultura Brasileira, 22:638-641.
Oliveira FL, Guerra JGM, Ribeiro RLD, Almeida DL, Silva EE, Urquiaga S \& Espindola JAA (2007) The use of sunn hemp as green manure intercropped with taro. Horticultura Brasileira, 25:562-566.

Oliveira FL, Araújo AP \& Guerra JGM (2011) Crescimento e acumulação de nutrientes em plantas de taro sob níveis de sombreamento artificial. Horticultura Brasileira, 29:292-298.

Puiatti M, Greeman S, Katsumoto R \& Favero C (1992) Crescimento e absorção de macronutrientes pelo inhame 'Chinês' e 'Japonês'. Horticultura Brasileira, 10:89-92.

Puiatti M, Fávero C, Finger FL \& Gomes JM (2000) Crescimento e produtividade de inhame e de milho doce em cultivo associado. Horticultura Brasileira, 18:24-30.

Puiatti M (2002) Manejo da cultura do taro. In: Carmo CAS (Ed.) Inhame e taro: sistema de produção familiar. Vitória, Incaper. p.203-254.

Rezende BLA, Cecílio Filho AB, Canato GHD \& Martins MIEG (2005) Análise econômica de consórcios de alface x tomate, em cultivo protegido, em Jaboticabal, SP. Científica, 33:42-49.

Rezende BLA, Cecílio Filho AB, Feltrim AL, Costa CC \& Barbosa JC (2006) Viabilidade da consorciação de pimentão com repolho, rúcula, alface e rabanete. Horticultura Brasileira, 25:36-41.

Rezende BLA (2008) Consórcios de pepino e alface em cultivo protegido: viabilidade agroeconômica. Tese de Doutorado. Universidade Estadual Paulista "Julio de Mesquita Filho", Jaboticabal. $163 \mathrm{p}$.

Rezende BLA, Cecílio Filho AB, Pôrto DRQ, Barros Junior AP, Silva GS, Barbosa JC \& Feltrim AL (2010) Consórcios da alface crespa e pepino em função da população do pepino e época de cultivo. Interciencia, 35:374-379.

Ribeiro CA, Guimarães PTG \& Alvares V VH (1999) Recomendações para uso de corretivos e fertilizantes em Minas Gerais: $5^{\text {a }}$ Aproximação. Viçosa, Comissão de Fertilidade do Solo do Estado de Minas Gerais. 359p.

SAEG (2007) Sistema para Análises Estatísticas e Genéticas. Versão 9.1. Viçosa, Fundação Arthur Bernardes. CD-ROM.

Sediyama MAN, Nascimento JLM, Lopes IPC, Lima PC \& Vidigal SM (2014) Tipos de poda em pepino dos grupos aodai, japonês e caipira. Horticultura Brasileira, 32:491-496.

Vieira JCB, Puiatti M, Cecon PR, Bhering AS, Silva GCC \& Colombo JN (2014) Viabilidade agroeconômica da consorciação do taro com feijão-vagem indeterminado em razão da época de plantio. Revista Ceres, 61:229-233. 